\title{
ASYMPTOTIC PRIME IDEALS RELATED TO DERIVED FUNCTORS
}

\author{
LEIF MELKERSSON AND PETER SCHENZEL
}

(Communicated by Louis J. Ratliff, Jr.)

\begin{abstract}
Let $R$ be a commutative noetherian ring. Let $N$ (resp. $A$ ) denote a noetherian (resp. artinian) $R$-module and $I$ an ideal of $R$. It is shown that for each integer $i$ the sets of prime ideals $\operatorname{Ass}_{R} \operatorname{Tor}_{i}^{R}\left(R / I^{n}, N\right)$ and $\operatorname{Att}_{R} \operatorname{Ext}_{R}^{i}\left(R / I^{n}, A\right), n=1,2, \ldots$, become for $n$ large independent of $n$.
\end{abstract}

\section{INTRODUCTION}

Let $R$ denote a commutative noetherian ring. For a noetherian $R$-module $N$ let $\operatorname{Ass}_{R} N$ denote its set of associated prime ideals; see, e.g., [4] for the definition. In a dual manner, one may define the set of attached prime ideals $\mathrm{Att}_{R} A$ for an artinian $R$-module $A$ (see [3] or the appendix to $\S 6$ in [4]). Let now $I$ be an ideal of $R$. The asymptotic behaviour of the sequences $\operatorname{Ass}_{R} N / I^{n} N$, resp. Att $A_{R} 0:_{A} I^{n}, n=1,2, \ldots$, has attained much attention. In [1] Brodmann has shown that the two sequences of associated prime ideals

$$
\operatorname{Ass}_{R} N / I^{n} N \text { and } \operatorname{Ass}_{R} I^{n-1} N / I^{n} N, \quad n=1,2, \ldots,
$$

become for large $n$ eventually constant. Dual to this result Sharp proved in [6] that the two sequences of attached prime ideals

$$
\operatorname{Att}_{R} 0:_{A} I^{n} \text { and } \operatorname{Att}_{R} 0:_{A} I^{n} / 0:_{A} I^{n-1}, \quad n=1,2, \ldots,
$$

become for large $n$ eventually constant. Now there are natural isomorphisms

$$
N / I^{n} N \cong R / I^{n} \otimes_{R} N \text { and } 0:_{A} I^{n} \cong \operatorname{Hom}_{R}\left(R / I^{n}, N\right)
$$

for each $n$. In the following we shall prove corresponding stability results for the associated, resp. attached, primes for the derived functors Tor ${ }_{i}$ of the tensor product, resp. $\mathrm{Ext}^{i}$ of the Hom-functor.

Theorem 1. For a given $i \geq 0$ the sequences of finite sets of associated prime ideals

$$
\operatorname{Ass}_{R} \operatorname{Tor}_{i}^{R}\left(R / I^{n}, N\right) \text { and } \operatorname{Ass}_{R} \operatorname{Tor}_{i}^{R}\left(I^{n-1} / I^{n}, N\right), \quad n=1,2, \ldots,
$$

become for large $n$ independent of $n$.

Received by the editors August 5, 1991.

1991 Mathematics Subject Classification. Primary 13A30; Secondary 13E05.

Key words and phrases. Associated prime, attached prime, asymptotic prime, Tor, Ext, Rees ring.

The second author was supported by a SERC Grant (No. GR /F/ 55584).

(C) 1993 American Mathematical Society $0002-9939 / 93 \$ 1.00+\$ .25$ per page 
Theorem 2. For a given $i \geq 0$ the sequences of finite sets of attached prime ideals $\operatorname{Att}_{R} \operatorname{Ext}_{R}^{i}\left(R / I^{n}, A\right)$ and $\operatorname{Att}_{R} \operatorname{Ext}_{R}^{i}\left(I^{n-1} / I^{n}, A\right), \quad n=1,2, \ldots$, become for large $n$ independent of $n$.

In the proof of Sharp's result (see [6]), it is not necessary to assume that $R$ is a noetherian ring. By a technique of Kirby (see [2]), it is possible to reduce the problem to the case of a finitely generated ideal. The authors conjecture that Theorem 2 is true also for a finitely generated ideal $I$ in an arbitrary commutative ring $R$.

On the other hand, it seems natural to ask for a uniform bound in Theorem 1 and Theorem 2, respectively. Namely, are the following sets of prime ideals

$$
\bigcup_{i \geq 0} \bigcup_{n \geq 1} \operatorname{Ass}_{R} \operatorname{Tor}_{i}^{R}\left(R / I^{n}, N\right) \text { and } \bigcup_{i \geq 0} \bigcup_{n \geq 1} \operatorname{Att}_{R} \operatorname{Ext}_{R}^{i}\left(R / I^{n}, A\right)
$$

finite? This seems to be an interesting question related to homological properties of $N$ and $A$, respectively. Moreover, is there a result corresponding to Theorem 1 for the sequences $\operatorname{Ass}_{R} \operatorname{Ext}_{R}^{i}\left(R / I^{n}, N\right), n=1,2, \ldots$ ?

In the following two sections we prove Theorems 1 and 2 , respectively. In the terminology we follow Matsumura's book [4].

\section{PROOF OF THEOREM 1}

Let $S=\bigoplus_{n \geq 0} S_{n}$ be a noetherian homogeneous $R$-algebra, i.e., $S=$ $R\left[a_{1}, \ldots, a_{l}\right]$, where $a_{i} \in S_{1}, i=1, \ldots, l$. For example $S$ could be the Rees algebra $R(I)$ with respect to the ideal $I$ of $R$.

2.1. Lemma. Let $X=\bigoplus_{n \in \mathbf{Z}} X_{n}$ be a finite graded $S$-module. Then for all large $n$

$$
\operatorname{Ass}_{R} X_{n}=\left\{P \cap R: P \in \text { Ass X, } S_{+} \not \subset P\right\},
$$

where $S_{+}$is the homogeneous ideal $\bigoplus_{n>0} S_{n}$ of $S$.

Proof (cf. [5, Theorem 3.1]). Consider the graded $S$-submodule $0:_{X}\left\langle S_{+}\right\rangle$of $X$ and the corresponding quotient module $\bar{X}=X / 0:_{X}\left\langle S_{+}\right\rangle$. Then

$$
\operatorname{Ass}_{S} \bar{X}=\left\{P \in \operatorname{Ass}_{S} X: S_{+} \not \subset P\right\} \quad \text { and } 0: \bar{X} S_{+}=0
$$

as is easily seen. Since $0:_{X}\left\langle S_{+}\right\rangle$is annihilated by some power of $S_{+}$, it is a finitely generated $R$-module. Therefore $\left(0:_{X}\left\langle S_{+}\right\rangle\right)_{n}=0$ for all large $n$, so $\bar{X}_{n} \cong X_{n}$ for $n$ large. With the above notation the maps

$$
\bar{X}_{n} \ni x \mapsto\left(a_{1} x, \ldots, a_{l} x\right) \in \bar{X}_{n+1}^{l}
$$

are injective for all $n$, note that $0: \bar{X}\left\langle S_{+}\right\rangle=0$, and consequently $\operatorname{Ass}_{R} \bar{X}_{n} \subset$ $\operatorname{Ass}_{R} \bar{X}_{n+1}$ for all $n$. By [5, Proposition 3.2] we have that $\operatorname{Ass}_{R} \bar{X}=\{P \cap R$ : $\left.P \in \operatorname{Ass}_{S} \bar{X}\right\}$. Now $\operatorname{Ass}_{R} \bar{X}=\bigcup_{n \in \mathbf{Z}} \operatorname{Ass}_{R} \bar{X}_{n}$, so the conclusion of the lemma follows.

2.2. Lemma. For each $i$ the sequence $\operatorname{Ass}_{R} \operatorname{Tor}_{i}^{R}\left(X_{n}, N\right), n \in \mathbf{Z}$, becomes eventually constant.

Proof. Note that $\operatorname{Tor}_{i}^{R}(X, N)$ can be given a structure of a finite graded $S$ module, whose component in degree $n$ is $R$-isomorphic to $\operatorname{Tor}_{i}^{R}\left(X_{n}, N\right)$. 
Applying Lemma 2.2 to $S=R(I)$ and $X=G(I)=\bigoplus_{n \geq 0} I^{n} / I^{n+1}$ we get the second assertion of Theorem 1. Furthermore if $0 \rightarrow X \rightarrow Y \rightarrow Z \rightarrow 0$ is an exact sequence of graded $S$-modules, where $X$ is assumed to be finite and $Y$ considered as an $R$-module is flat, then $\operatorname{Tor}_{i}^{R}(Z, N)$ is a finite $S$-module for all $i \geq 1$. Namely, from the exactness of $0 \rightarrow X \rightarrow Y \rightarrow Z \rightarrow 0$ and the $R$-flatness of $Y$ it follows that

$$
\begin{aligned}
& \operatorname{Tor}_{i}^{R}(Z, N) \cong \operatorname{Tor}_{i-1}^{R}(X, N) \quad \text { for } i \geq 2, \\
& \operatorname{Tor}_{1}^{R}(Z, N) \cong \operatorname{Ker}\left(X \otimes_{R} N \rightarrow Y \otimes_{R} N\right),
\end{aligned}
$$

which is a graded submodule of the finite graded $S$-module $X \otimes_{R} N$. Considering now $S=R(I)$ and the exact sequence $0 \rightarrow R(I) \hookrightarrow R[t] \rightarrow R[t] / R(I) \rightarrow 0$ we get the first assertion of Theorem 1, so the proof of Theorem 1 is now complete.

\section{Proof of Theorem 2}

3.1. Lemma. Let $S=R\left[a_{1}, \ldots, a_{l}\right]$ be a graded $R$-algebra, where $a_{i} \in S_{1}$, $i=1, \ldots, l$. Let $Y$ be a graded artinian $S$-module. Then

$$
\operatorname{Att}_{R} Y_{-n}=\left\{P \cap R: P \in \operatorname{Att}_{S} Y, S_{+} \not \subset P\right\}
$$

for all large $n$.

Proof. Let $\bar{Y}=\left\langle S_{+}\right\rangle Y$ be the graded $S$-submodule $\bigcap_{n \geq 1}\left(S_{+}\right)^{n} Y$ of $Y$, which is equal to $\left(S_{+}\right)^{n} Y$ for $n$ large. Note that

$$
\operatorname{Att}_{S} \bar{Y}=\left\{P \in \operatorname{Att}_{S} Y: S_{+} \not \subset P\right\} ;
$$

see [3, (3.3)]. Since $Y / \bar{Y}$ is an artinian graded $S$-module annihilated by $\left(S_{+}\right)^{n}$ for some $n$, it is artinian also as an $R$-module, so $\bar{Y}_{-n} \cong Y_{-n}$ for all large $n$. Furthermore $\bar{Y}=S_{+} \bar{Y}$, so the argument given in the proof of [5, Theorem 4.3] shows that $\operatorname{Att}_{R} \bar{Y}_{-n}$ is for large $n$ equal to $\left\{P \cap R: P \in \operatorname{Att}_{S} \bar{Y}\right\}$.

Remark. As it follows from the proof, it is not necessary to assume $R$ to be noetherian in Lemma 3.1.

For a graded $S$-module $X$ put

$$
H(X, A)=\bigoplus_{n \in \mathbf{Z}} \operatorname{Hom}_{R}\left(X_{-n}, A\right),
$$

which is in a natural way a graded $S$-module. The multiplication map $S_{m} \times$ $X_{-(m+n)} \rightarrow X_{-n}$ induces the multiplication map

$$
S_{m} \times \operatorname{Hom}_{R}\left(X_{-n}, A\right) \rightarrow \operatorname{Hom}_{R}\left(X_{-(m+n)}, A\right) .
$$

If $X$ is a finite $S$-module, $H=H(X, A)$ is an artinian $S$-module. To prove this, use [5, Corollary 2.2]. One has only to note that from $S_{1} X_{n}=X_{n+1}$ for all large $n$ it follows that $\left(0:_{H} S_{+}\right)_{n}=0$ for all but finitely many $n$.

In a minimal resolution $0 \rightarrow A \rightarrow E_{A}^{0} \rightarrow E_{A}^{1} \rightarrow \cdots$ of the artinian $R$ module $A$, the modules $E_{A}^{i}, i=0,1, \ldots$, are all artinian. This implies that for each $i$ there is an artinian graded $S$-module $E^{i}(X, A)$, namely a certain subquotient of $H\left(X, E_{A}^{i}\right)$, whose component in degree $n$ is $R$-isomorphic to $\operatorname{Ext}_{R}^{i}\left(X_{-n}, A\right)$. From Lemma 3.1 and the above discussion we get: 
3.2. Corollary. If $X$ is a finite graded $S$-module, then for each $i$ the sequence

$$
\operatorname{Att}_{R} \operatorname{Ext}_{R}^{i}\left(X_{n}, A\right), \quad n \in \mathbf{Z},
$$

becomes eventually constant.

Applying Corollary 3.2 to $S=R(I)$ and $X=G(I)$ the second assertion of Theorem 2 now follows. Furthermore if we have an exact sequence $0 \rightarrow X \rightarrow$ $Y \rightarrow Z \rightarrow 0$ of graded $S$-modules, where $X$ is supposed to be finite and $Y$ considered as an $R$-module is projective, we have

$$
\begin{aligned}
& E^{i}(Z, A) \cong E^{i-1}(X, A) \text { for } i \geq 2, \\
& E^{1}(Z, A) \cong \operatorname{Coker}(H(Y, A) \rightarrow H(X, A)) .
\end{aligned}
$$

Therefore $E^{i}(Z, A)$ is for each $i \geq 1$ an artinian graded $S$-module. By Lemma 3.1 for each $i \geq 1$ the sequence $\operatorname{Att}_{R} \operatorname{Ext}_{R}^{i}\left(Z_{n}, A\right), n \in \mathbf{Z}$, becomes eventually constant. Applying this to $S=R(I)$ and the exact sequence $0 \rightarrow R(I) \hookrightarrow$ $R[t] \rightarrow R[t] / R(I) \rightarrow 0$ we obtain the first assertion of Theorem 2. The case $i=0$ is known, since $\operatorname{Hom}_{R}\left(R / I^{n}, A\right) \cong 0:_{A} I^{n}$ (see, e.g., [6]). The proof of Theorem 2 is now complete.

\section{REFERENCES}

1. M. Brodmann, Asymptotic stability of $\operatorname{Ass}\left(M / I^{n} M\right)$, Proc. Amer. Math. Soc. 74 (1979), 16-18.

2. D. Kirby, Artinian modules and Hilbert polynomials, Quart. J. Math. Oxford (2) 24 (1973), 47-57.

3. I. G. Macdonald, Secondary representations of modules over a commutative ring, Sympos. Math., vol. 11, Academic Press, London and New York, 1973, pp. 23-43.

4. H. Matsumura, Commmutative ring theory, Cambridge Univ. Press, Cambridge, 1986.

5. L. Melkersson, On asymptotic stability for sets of prime ideals connected with the powers of an ideal, Math. Proc. Cambridge Philos. Soc. 107 (1990), 267-271.

6. R. Y. Sharp, Asymptotic behaviour of certain sets of attached prime ideals, J. London Math. Soc. (2) 34 (1986), 212-218.

Department of Mathematics, University of Lund, Box 118, S - 22100 Lund, Sweden

FACHBereich Mathematik und INFormatik, Martin-Luther-Universität Halle-WitTenberg, Postfach, O - 4010 Halle, Germany 